Sedó Masís, P. (2021). EL TAMAL Y LA TRADICIÓN TAMALERA EN COSTA RICA. Revista Herencia, Vol. 34 (2), enerojunio, 136-164.

\title{
EL TAMAL Y LA TRADICIÓN TAMALERA EN COSTA RICA
}

\author{
The tamale and the "tamalera" tradition in Costa Rica
}

Recibido: $12-01-2021$

Aprobado: 25-03-2021

\author{
Patricia Sedó Masís \\ Universidad de Costa Rica, Costa Rica \\ patricia.sedo@ucr.ac.cr
}

\begin{abstract}
Patricia Sedó Masis es Docente catedrática de la Escuela de Nutrición de la Universidad de Costa Rica, desde 1989, con dedicación al estudio de la cultura alimentaria y la nutrición geriátrica. Posee una Maestría en Gerontología de la UCR y una Maestría en Administración Educativa de la Universidad Latina de Costa Rica. Premio de Acción Social por el Área de Salud 2020.
\end{abstract}

\section{RESUMEN}

Este trabajo presenta una descripción de aspectos socioculturales, gastronómicos y nutricionales del tamal en Costa Rica, a partir de una revisión documental y 50 entrevistas realizadas a personas portadoras de la tradición, durante el 2014 y 2019. La tradición tamalera reúne un conjunto de saberes y prácticas de alto valor cultural, socio productivo y nutricional. Hay diferencias según ingredientes, procedimientos, nombres dados en las localidades, así como en envolturas y acompañamientos en la mesa. Trasciende la necesidad de alimentarse, y otorga un sentido de pertenencia e identidad. La complejidad en su elaboración, la percepción negativa sobre su composición, la pérdida en la herencia familiar de las recetas, la mayor oferta comercial de tamales y el cambio alimentario con predilección por otros productos, figuran entre los aspectos que más afectan la tradición. No obstante, se valora como un alimento artesanal que conserva los sabores familiares e insustituibles. Este trabajo constituye un insumo para el desarrollo de estrategias integrales que contribuyan con la conservación y difusión del patrimonio alimentario del país.

Palabras clave: tamal; cocina costarricense; cultural alimentaria; maíz; alimentación tradicional.

\section{ABSTRACT}

This study presents a description of the sociocultural, gastronomics and nutritional aspects of the tamale in Costa Rica, based on a documentary review and 50 interviews carried out with people carrying the tradition, during 2014 and 2019. The tamalera tradition brings together a set of knowledge and practices of high cultural, productive and nutritional value. There are differences according to ingredients, procedures, names given in the localities, as well as in wraps and accompaniments at the table. It transcends the need to feed, and grants a sense of belonging and identity. The complexity in its preparation, the negative perception of its composition, the loss of the family inheritance of the recipes, the greater commercial offer of tamales and the food change with a predilection for other products, are among the aspects that most affect the tradition. However, it is valued as an artisanal food that preserves the familiar and irreplaceable flavors. This study constitutes an input for the development of comprehensive strategies that contribute to the conservation and dissemination of the country's food heritage. 
Revista Herencia, Vol. 34 (2), enero-junio, 2021.

Keywords: tamal; Costa Rican food; culture food; corn; tradicional food.

\section{Introducción}

Entre la diversidad de alimentos tradicionales de Costa Rica, el tamal ocupa un lugar especial. Su origen se remonta a unos cuatro mil años en civilizaciones de México y norte de Centroamérica y, por el intercambio cultural y comercial, se difundió al resto del continente (Vela, 2017).

La palabra "tamal", del náhuatl tamalli, significa "envuelto" (Jara, 1988, p. 118). De acuerdo con Sánchez (2006, p. 193), este concepto tiene un amplio uso en América, y comprende Estados Unidos, México, Centroamérica, Cuba y América del Sur. En México se dispone de cientos de tamales que se diferencian según los nombres dados, su preparación y usos; mientras que en Centroamérica y América del Sur reciben nombres tales como bollos, chuchitos, paches, nacatamales (del azteca nacatl "carne" y tamalli "envuelto"), yoles y yoltamales (del azteca yoyotl “corazón” y tamalli “envuelto), hallacas o hayacas, humitas o humintas, entre otros (Vela, 2017; Ramos, 2014).

La tradición tamalera ha sobrevivido a pesar de los cambios alimentarios que ha enfrentado la población, y el menor uso del maíz en la actualidad. El presente trabajo tiene como objetivo analizar aspectos socioculturales, gastronómicos y nutricionales del tamal, y la tradición tamalera en Costa Rica.

\section{Método de trabajo}

Se procedió a una revisión documental tomando como base recetarios y otros documentos sobre historia y nutrición; asimismo, se realizaron 50 entrevistas semiestructuradas a personas portadoras de la tradición en Aserrí, Atenas, Nicoya, Puriscal, Santa Ana, Santa Cruz de Guanacaste, Turrialba, Tierra Blanca de Cartago y Zarcero, entre el 2014 y 2019. También se hizo una revisión de publicaciones en redes 
Revista Herencia, Vol. 34 (2), enero-junio, 2021.

sociales de la Caja Costarricense de Seguro Social (CCSS) entre octubre y diciembre, del 2014 al 2020.

Este estudio forma parte del Programa EC-436 "Alimentos tradicionales y cocina tradicional de Costa Rica: aportes para contribuir con su salvaguardia" de la Escuela de Nutrición de la Universidad de Costa Rica e inscrito en la Vicerrectoría de Acción Social.

\section{El maíz y el tamal en la cocina prehispánica}

El maíz es una planta gramínea que constituyó la base de la alimentación de los pueblos prehispánicos en Mesoamérica, junto con el frijol, diversidad de ayotes y otros alimentos disponibles en el medio. Su nombre náhuatl es tlallotli que significa "corazón de la tierra"; mientras que maíz es voz del taíno mahis que significa "lo que sustenta la vida" (Merlo et al., 2016).

Existe evidencia arqueológica en relación con utensilios, espacios dedicados a la cocina, restos de alimentos, y estudios sobre alimentación y lingüística que muestran la importancia del tamal para las poblaciones precolombinas. Este alimento fue básico para la población, presente en rituales y festividades, y asociado con abundancia, prosperidad y energía (Vela, 2017).

El cronista Fray Bernardino de Sahagún, en su obra llamada la "Historia natural de las cosas de la Nueva España", escrita en el siglo XVI, realizó una descripción detallada de la alimentación de las poblaciones indígenas, y refirió el máximo aprovechamiento de los recursos alimentarios del medio y el valor del maíz, tal como se refleja en la siguiente cita:

Y al tiempo que el maizal estaba creciendo y empezaba a dar mazorcas comenzaban luego a coger de las menores para comer o para comprar carne o pescado y el vino de la tierra para beber (el pulque), y de lo mismo servían las calabazas y los chiles verdes que se daban en el tiempo de verano y cuando el maíz ya estaba sazonado gustaban lo que podían de las mazorcas grandes para comprar 
Revista Herencia, Vol. 34 (2), enero-junio, 2021.

con ellas lo que había menester y para comerlas cocidas y hacer de ellas tortillas y tamales y así al tiempo de cosechar no cogían sino un poco por haberlo gastado y comido antes de que sazonase, luego que había cogido lo poco, compraban gallinas y perrillos para comer y hacían banquetes convidándose unos a otros (de Sahagún, 1989, p. 162).

En el complejo sistema agrícola, principalmente la milpa, se refleja un universo de elementos religiosos, económicos, sociales y alimentarios que favorecieron la evolución de los pueblos en Mesoamérica. La importancia que tenían los alimentos, entre ellos el maíz y sus derivados, no sólo se relacionaba con el sustento, sino que obsequiarlos y consumirlos en grupo formaba parte del ritual, y era un halago para quien los recibía (Ramos, 2014; Vela, 2017).

El tamal sobresale como un alimento presente en la vida cotidiana y ceremonial, y mostró variedad en formas de preparación y usos, según jerarquía o actividad festiva. Los tamales sin relleno eran de consumo popular; mientras que aquellos con rellenos diversos eran consumidos por personas de mayor estatus o se usaban como ofrendas en festividades religiosas (Vela, 2017).

Como parte del relleno se incluían carne de aves, anfibios, pescado, camarones, perro, caracoles, semillas de ayote y otras cucurbitáceas, diferentes hierbas, chiles, tomate y otros. Todos estos alimentos eran propios de la región, por lo que eran fáciles de obtener en zonas boscosas, ríos y mares (Bustamante, 1829; Vela, 2017; Ross, 1990).

El cronista Fernández de Oviedo, en su obra "Sumario de la natural historia de las Indias" escrita en el siglo XVI, realizó una detallada descripción del tamal, su vida útil y conservación, tal como se describe seguidamente:

Las indias especialmente lo muelen en una piedra algo concavada, con otra redonda que en las manos traen, a fuerza de brazos, como suelen los pintores moler las colores, y echando de poco poca agua, la cual así moliendo se mezcla con maíz, y sale de allí una manera de pasta como masa, y toman de aquello que envuélvenlo en una hoja de yerba, que ya ellos tienen para esto, o en una 
Revista Herencia, Vol. 34 (2), enero-junio, 2021.

hoja de la caña del propio maíz o otra semejante, y échanlo en las brasas, y ásase, y endurécese, y tórnase como pan blanco y hace su corteza por desuso, y de dentro de este bollo está la miga algo más tierna que la corteza; y hase de comer caliente, porque estando frío, ni tiene tan buen sabor ni es tan bueno de mascar, porque está más seco y áspero. También estos bollos se cuecen, pero no tienen tan buen gusto; y este pan, después de cocido o asado, no se sostiene sino muy pocos días, y luego, desde a cuatro o cinco días, se mohece y no está de comer (Fernández de Oviedo, 1950, p. 94-95).

En el período de conquista y Colonia se presenta un gran choque cultural, y el tamal fue perdiendo, en parte, su valor simbólico y ritual mostrado en la prehispanidad. No obstante, ha permanecido su vínculo con fiestas familiares y comunitarias (Vela, 2017).

De conformidad con la Oficina Nacional de Semillas (s.f.), en Costa Rica existe evidencia arqueológica del maíz desde hace más de 5600 años. Resalta la importancia del cultivo y su impacto en el desarrollo económico y social de la población; no obstante, a inicios de la década de 1980, se presenta una fuerte crisis económica y cambios en el modelo de desarrollo que trajo el fortalecimiento de los tratados de libre comercio, y la mayor importación del grano. Ello generó una afectación directa a pequeños agricultores, lo cual unido a cambios alimentarios en la población, llevaron a la merma en la producción local, y el menor consumo de maíz.

\section{Descripción general del tamal y sus formas de preparación}

El uso del vocablo "tamal" en Costa Rica aplica al producto elaborado con una masa, principalmente de maíz, y envuelto en hojas. También se usa para nombrar otras preparaciones tipo pasteles dulces a base de maíz u otros productos harinosos, generalmente horneados. Entre ellos se encuentran el tamal de elote tierno molido, el tamal asado a base de masa de maíz y derivados lácteos, así como el de yuca o maicena (almidón de maíz). 
Revista Herencia, Vol. 34 (2), enero-junio, 2021.

Como parte de los resultados, se obtuvo la descripción de más de cien preparaciones, con variantes en cuanto a tipos y proporción de ingredientes, envolturas, formas de cocción y nombres dados a los productos. En el siguiente cuadro se presenta una clasificación general de los tipos de tamales según forma de preparación:

\begin{tabular}{|c|c|}
\hline Tipos de tamales & Descripción general \\
\hline $\begin{array}{l}\text { Tamales envueltos en } \\
\text { hojas y cocidos al vapor o } \\
\text { hervido }\end{array}$ & 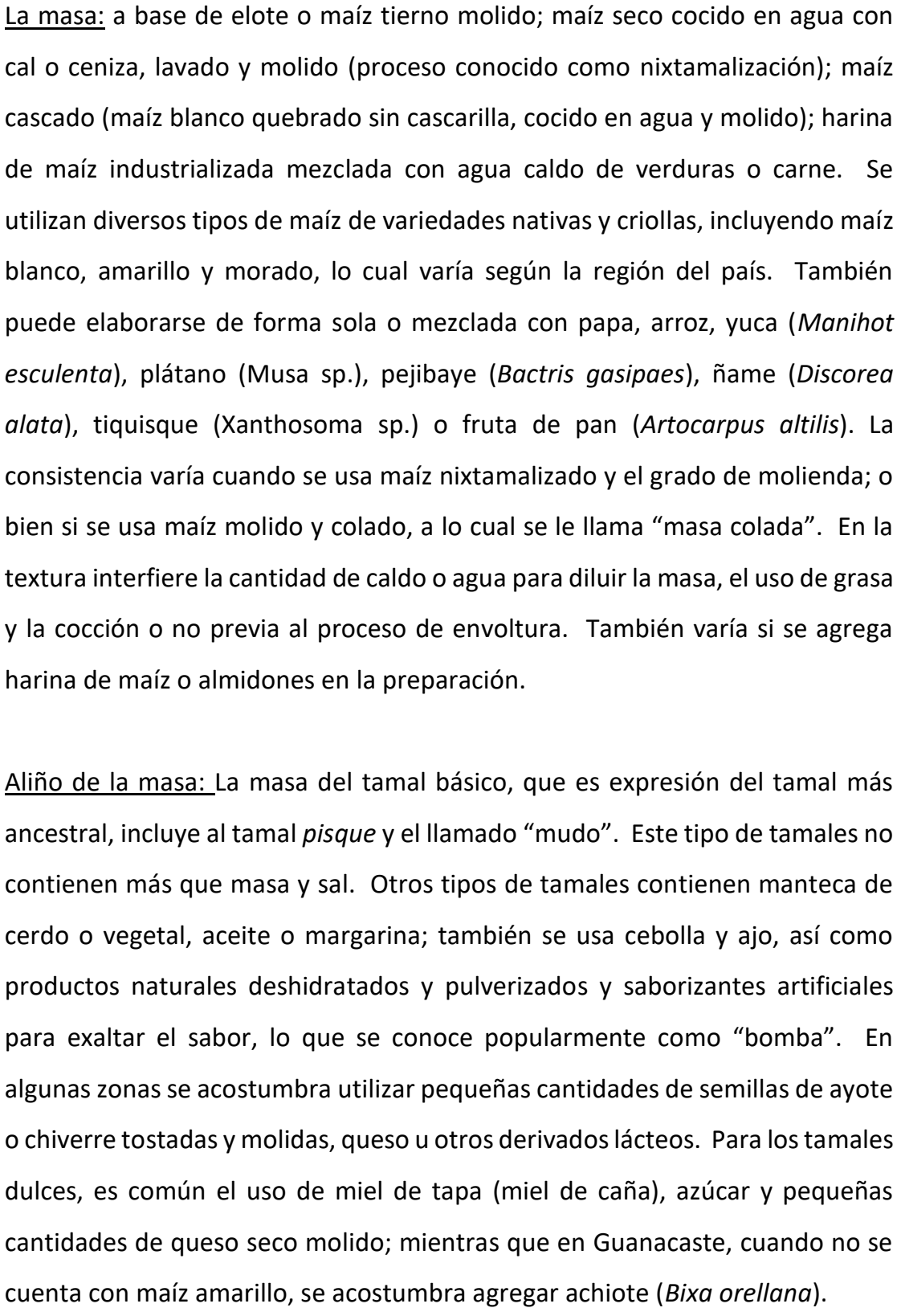 \\
\hline
\end{tabular}


Revista Herencia, Vol. 34 (2), enero-junio, 2021.

Rellenos: el pipián es una mezcla de la misma masa con manteca y achiote, a la que puede agregarse tomates, chiles y/o semillas tostadas, lo cual corresponde a una receta de las más antiguas. También puede agregarse chicharrón molido, cebolla y ajo. El pipián es sustituido por arroz con achiote. En el caso de carnes, se utiliza sola o combinada la carne de cerdo (posta, costilla, chorizo y/o tocino) y pechuga de pollo. También se utilizan picadillos de papa con o sin carne y/o frijoles blancos; de vainicas con zanahoria; hojas amargas como chicasquil (Cnidoscolus aconitifolius), zorrillo (Cestrum recemosum) o mostaza criolla (de la familia Brassicae) mezcladas con papa o la piel del cerdo (pellejo). Es usual incluir vegetales en trozos pequeños (rodajas, gajos o cuadritos) tales como zanahoria, papa, vainica, chile dulce y chayote; granos (arvejas y garbanzos) e hierbas aromáticas como culantro castilla (Coriandum sativum) o coyote (Erygium foetidum). La forma, el grosor, tamaño y colocación en la masa varía según la tradición familiar. En menor frecuencia se usa huevo duro, aceitunas, alcaparras, ciruelas y uva pasa, tomate, encurtido de mostaza, atún y sardina. En el caso de tamales dulces, se usan rellenos a base de ciruelas o uvas pasa, queso molido con tapa de dulce, conserva de chiverre, mermeladas de frutas, dulce de leche, entre otros.

Envolturas: las más comunes son las hojas de la mazorca frescas o secas hidratadas, variedad de hojas de musáceas soasadas y hojas de caña de maíz frescas. El tamal de arroz que se elabora en la zona sur del país se envuelve en la hoja de bijagua (Calathea lutea) o platanilla (Heliconia sp.). En Guanacaste también se usan las hojas de espavel (Anacardium excelsum) para el tamal pisque, las cuales son de grandes dimensiones y son comestibles cuando están tiernas. En cuanto al tamaño del tamal, éste varía según la cantidad de masa y relleno. Los tamales que se producen en Guanacaste son de mayor tamaño respecto a los del resto del país; el peso de la masa puede alcanzar hasta los 300 gramos. En el caso del Valle Ventral, el peso de la masa puede oscilar entre 125 y 200 gramos, siendo más comunes los de "masa colada". Se llama "tamalito" o "tamalito de boca" el de dimensiones pequeñas cuya cantidad de masa no supera los 50 gramos. Las formas varían según la región, localidad y familia. Los hay cuadrados, rectangulares, aplanados o de formas más abultadas. Existen también los de forma cilíndrica o gema, a base de solo masa o rellenos con frijoles molidos o picadillo, los cuales se parten en rodajas en el 
Revista Herencia, Vol. 34 (2), enero-junio, 2021.

\begin{tabular}{|c|c|}
\hline & $\begin{array}{l}\text { momento del consumo, y se sirven fríos o calientes, y son conocidos como } \\
\text { "tamales mudos". } \\
\text { El amarrado: generalmente se amarran de dos en dos, conocido popularmente } \\
\text { como "piña". Se procura que los extremos queden bien doblados para evitar } \\
\text { derrames de contenido o la entrada de agua al interior en el momento de la } \\
\text { cocción. Tamales de grandes dimensiones se amarran solos. En algunas partes } \\
\text { de Guanacaste se observó que no se amarran los tamales, dado que la masa es } \\
\text { más compacta, y se cuecen al vapor. Se colocan de forma circular en la olla, } \\
\text { con lo cual se logra un buen prensado que evita que la entrada de agua. } \\
\text { La cocción: se utilizan grandes ollas o pailas. La cocción a la leña es la más } \\
\text { común, no solo por facilidad, sino porque el sabor a humo es altamente } \\
\text { valorado por las personas. El hervido es el método más común, aunque } \\
\text { también en Guanacaste se recurre a la cocción al vapor, con la colocación de } \\
\text { un enrejado con ramas secas en el interior de la olla, cubierto con hojas de } \\
\text { plátano, y encima se colocan los tamales. }\end{array}$ \\
\hline Tamales horneados & $\begin{array}{l}\text { La masa: a base de elote tierno, masa de maíz o productos harinosos molidos } \\
\text { molidos en forma de puré, tales como yuca cruda rallada o cocida, tiquisque, } \\
\text { ñame, pejibaye, arroz cocido con leche, camote (Ipomoea batatas) o ayote } \\
\text { sazón (Cucurbita moschata). También son comunes las harinas industrializadas } \\
\text { tales como harina de maíz, almidón de maíz, de plátano, entre otras. } \\
\text { Aliño de la masa: según el tipo de tamal se usa manteca de cerdo o vegetal, } \\
\text { margarina o mantequilla, natilla, leche agria, quesos variados, coco, azúcar o } \\
\text { tapa de dulce. Se utiliza huevo y un agente leudante para lograr una textura } \\
\text { suave y esponjosa. En cuanto a saborizantes, es común el uso de esencia de } \\
\text { vainilla, canela, clavo de olor y nuez moscada; además, ralladura de naranja o } \\
\text { limón y licores. } \\
\text { Forma de cocción: las masas se vierten en bandejas previamente engrasadas o } \\
\text { cubiertas con hoja de plátano. Cuando se asa en brasas calientes se llama } \\
\text { "tamal a las brasas" o "tamal encenizado", y es una de las formas de cocción }\end{array}$ \\
\hline
\end{tabular}


Revista Herencia, Vol. 34 (2), enero-junio, 2021.

más antiguas. También se cuecen en hornos de barro, ollas (tamal de olla), y en hornos convencionales de gas o eléctricos.

Cuadro 1. Descripción general de tamales en Costa Rica, según ingredientes

y formas de preparación.

Fuente: Elaboración propia.

En ampliación a lo indicado en el cuadro anterior, el tamal más simple, sustituto de la tortilla, es aquel elaborado únicamente con masa de maíz. Puede elaborarse todo el año, aunque es en Semana Santa cuando está más presente en la mesa familiar, dada la tradición de consumir productos sin carne. El tamal pisque, propio de la cocina de Guanacaste, podría considerarse como una de las formas de preparación más antiguas, heredada de la cocina prehispánica. En el Valle Central existe otro tipo de tamal a base de masa sola o con relleno de frijoles molidos o picadillo de papa, llamado "tamal mudo".

Los tamales a base de elote y envueltos, preferiblemente, en hoja de maíz o tusa forman parte de las preparaciones más antiguas. En este caso, la masa es exclusivamente de elote fresco molido. Como parte de los cambios de la receta a lo largo del tiempo, se evidencia el agregado de sal o dulce, así como queso, natilla o margarinas e inclusive huevo. En Guanacaste se preparan los yoles o yoltamales con maíz tierno amarillo o morado tierno, con recetas que varían respecto a las seguidas en el Valle Central.

En relación con la población indígena costarricense boruca, destaca el uso del término "bollo" para referirse a los tamales elaborados con maíz tierno o masa de maíz nixtamalizada (Garro, 2010).

Los "tamalitos", al utilizarse generalmente como un aperitivo en ocasiones especiales, presentan mayor variedad en cuanto a rellenos. Sobresale el uso de frijoles negros o rojos molidos, picadillos de papa con carne u hojas, o a base de vainicas y zanahoria finamente picadas. También los hay de mermeladas coloridas y sabor agridulce, como fresa o mora. 
Revista Herencia, Vol. 34 (2), enero-junio, 2021.

Entre los rellenos dulces más comunes están el queso, azúcar o dulce raspado, pasas y especias tales como canela, clavo de olor, vainilla o nuez moscada y cacao. Estos rellenos son utilizados en masas de arroz, maíz o plátano maduro. Los considerados por las personas entrevistadas como "tamales más tradicionales" o "criollos", se describen como aquellos con masa de "puro maíz"; es decir, que no incluye harinas industrializadas u otros productos. Como parte del relleno se cita el pipián o el arroz con achiote, carne de cerdo o pollo, zanahoria, arvejas tiernas y/o garbanzos, vainicas y chile dulce. Se considera que ésta es la receta más popular, misma que está al alcance de la mayoría de la población. Los tamales que incluyen aceitunas, alcaparras o pasas, algunos los califican como preparaciones foráneas o gourmet, no considerados como parte de la cocina tradicional del país.

También sobresale la mezcla del maíz con plátano o banano verde o maduro, yuca, papa y otras raíces o tubérculos, algo común en comunidades de Puntarenas, hacia el norte y sur del país, y Cartago. Los tamales a base de pejibaye son comunes en Tucurrique.

Respecto a la innovación, resalta la oferta de nuevos tipos de tamales en restaurantes. Así, por ejemplo, en negocios de cocina caribeña se ofrecen tamales donde la masa es aliñada con leche de coco e incluyen rellenos tales como rice \& beans o pescado. Los tamales "dietéticos" o "vegetarianos" se caracterizan por un bajo contenido de grasa y rellenos tales como berenjena, hongos, texturizados de soya, pescado, entre otros. Esta es una tendencia que cada día cobra más fuerza, en seguimiento a ciertos regímenes dietéticos.

Todo lo anterior es muestra de la versatilidad del tamal, y la convergencia de sabores propios de las cocinas de las diversas regiones del país, así como la adaptación de las recetas según las necesidades de las personas.

\section{El valor simbólico del tamal}


Revista Herencia, Vol. 34 (2), enero-junio, 2021.

El tamal está presente en la alimentación cotidiana, así como en eventos familiares y comunitarios. Cuando se le pregunta a las personas sobre el mismo, se asocia con ambientes festivos, alegría, comensalidad y reunión familiar.

Existe una relación directa entre el consumo y el valor que las personas le otorgan, el cual va más allá de la dimensión biológica, puesto que el tamal forma parte de la biografía colectiva, tal como lo indican Bourges \& Vargas (2015): “estar ante un tamal de elote no es solamente cuestión de consumir fibra, ácidos grasos, proteína o energía, sino que llega a tener un tinte más profundo si evoca el efecto de la abuela o la experiencia de un viaje" (p.4).

Una persona entrevistada afirmó lo siguiente: "podrían venir pestes, terremotos, pero una tamaleada no puede faltar en mi casa. Es algo como que forma parte nuestra...no podemos fallar, porque es un compromiso con los que nos han dejado [...] es un legado (F. Cubero, comunicación personal, 14 de marzo de 2018).

Los tamales se asocian con herencia y reunión familiar, comida tradicional y fiesta. Sobre este tema indicó una persona: “¿cuál tico no conoce qué es un tamal? Es como no saber qué es un gallo pinto o que nuestro país tiene siete provincias [...] El tamal forma parte muy nuestra, es alto totalmente costarricense" (R. Rodríguez, comunicación personal, 21 de octubre de 2016).

Este alimento evoca sabores de la infancia y herencia familiar, señalado por una persona de la siguiente manera:

Comer los tamales que hace mi mamá es como estar al lado de mis abuelas y tías, cuando era niña y me subía al taburete para alcanzar el borde de la gran mesa de madera, y observar detenidamente cómo ellas los elaboraban...llenos sabor y color, uno a uno, con mucho cuidado y paciencia... Es un sabor incomparable, muy especial, que no lo encuentro en un tamal comercial, porque ese tamal 
Revista Herencia, Vol. 34 (2), enero-junio, 2021.

no sólo envuelve la masa, sino también el amor, el calor de hogar que encuentro en cada bocado que me llevo a la boca, y me llena el estómago y el corazón (S. Jiménez, comunicación personal, 13 de diciembre de 2019).

Se muestra así el vínculo entre el alimento con las emociones, y el valor del intercambio intergeneracional en relación con saberes y prácticas culinarias familiares que se heredan de generación en generación.

En época navideña, el consumo de tamales de cerdo es más frecuente que otros. Para una gran mayoría de personas entrevistadas se considera natural comerlo en varios tiempos de comida durante un día, sin que se muestre aburrimiento o empacho. El alto consumo forma parte de las actividades festivas que se disfrutan en este tiempo, y que rompen con la rutina de alimentación seguida durante el resto del año. Tan común es el consumo de tamales de cerdo en diciembre, que una gran mayoría los llaman "tamales navideños".

El tiempo dedicado a la preparación de los tamales y su consumo se conoce popularmente como "tamaleada". Este término se refiere a la reunión grupal donde se motiva al intercambio de recetas y tamales entre familias. Se presenta un amplio flujo de información que contribuye a mantener viva la tradición, y a su vez se favorece la comensalidad y la socialización.

Una vez finalizada la cocción, lo más esperado es probar y repartir los tamales. Es una actividad muy disfrutada por las personas encargadas de la elaboración, pero también por las personas convidadas. No hay mejor recompensa, luego de un arduo trabajo, que sentarse a la mesa para comerse el tamal, y brindar o recibir la opinión sobre el resultado final.

\section{Otros aspectos culinarios y nutricionales del tamal}


Revista Herencia, Vol. 34 (2), enero-junio, 2021.

Para preparar tamales se requiere conocimiento, práctica y apoyo de otros. Se requiere del liderazgo de una persona experta, principalmente mujeres de avanzada edad conocedoras de la tradición, que guíen el proceso para obtener buenos resultados.

Se presenta una jerarquía en la distribución del trabajo. Las tareas se reparten entre las personas participantes, sean miembros de la familia o invitados, con el establecimiento de flujos de producción eficientes, y un máximo aprovechamiento del espacio de la cocina, incluyendo hasta el solar de la casa. La familia puede dedicar hasta un día completo, y de forma exclusiva, a la preparación de tamales.

La jerarquía se asocia con el nivel de complejidad de las tareas, y existen diferencias según género y edad. La persona de mayor jerarquía, generalmente mujer, es la encargada de la elaboración de la masa y las carnes; a los hombres les corresponde labores con esfuerzo físico, como la instalación de fogones, cocción y molienda del maíz, conseguir las hojas o pelado de verduras. Estas actividades pueden ser compartidas con otros miembros menos experimentados en la cocina o de menor edad. Las personas de la envoltura son las responsables de asegurar que el producto esté bien presentado, y otorgan el sello distintivo familiar respecto a la forma del tamal.

De la masa existen sus secretos en la elaboración, tal como lo refiere la frase popular el secreto de un buen tamal está en su masa.

Sobresale un proceso tecnológico ancestral, donde el maíz es cocido en agua con cal y ceniza, al que se llama "nixtamalización". Se considera que la técnica es aplicada desde el Preclásico Medio, cuya invención representa un hito en la alimentación. Lo anterior, dado que permitió mejorar las reservas alimentarias, y disponer del grano seco a lo largo del año, que se podía cocer y transformar en masa, lo que dio origen a las tortillas y bollos envueltos o tamales (Vela, 2017; Merlo et al., 2016).

La palabra náhuatl nixtayol, utilizada en Guanacaste para referirse al maíz cocido, proviene de nextli "ceniza” y tlayolli "maíz seco desgranado" (Jara, 1988, p.117). Por su parte, en la 
Revista Herencia, Vol. 34 (2), enero-junio, 2021.

población indígena boruca, al maíz nixtamalizado con ceniza se llama estallol (Garro, 2010, p. 109).

El nixtamal, nixtayol o nesqueza (del náhuatl nextli "ceniza" e izquitil "maíz") consiste en cocinar el maíz en un medio alcalino: 1 kilogramo de maíz por dos a tres litros de agua mezclada con ceniza y/o cal. Se cocina de 30 a 60 minutos, luego se deja en remojo de 12 a 14 horas y, posteriormente, se aplican varias lavadas con agua fría para eliminar el hollejo y otros residuos, y luego proceder con la molienda.

La ceniza era el principal producto para alcalinizar el agua de cocción de maíz en la época precolombina, a la cual también se agregaban conchas molidas como fuente de sales de calcio; luego comenzó a usarse polvo de piedra caliza (cal) (Vela, 2017). Este proceso aún es aplicado en la actualidad, incluso a nivel industrial (Castillo et al., 2009; Billeb de Sinibaldi \& Bressani, 2001).

En la nixtamalización se producen cambios químicos, estructurales y nutricionales importantes. Su invención fue altamente favorable para la nutrición de las poblaciones precolombinas, ya que se mejora la biodisponibilidad de la niacina, lo cual evita la enfermedad conocida como Pelagra; además, se incrementa la biodisponibilidad de aminoácidos esenciales. Al utilizarse la ceniza y/o cal se logra un enriquecimiento de la masa con minerales, principalmente calcio, magnesio y fósforo (Castillo et al., 2009).

Se puede usar la "masa cruda"; es decir, aquella que una vez cocido el maíz, se muele o se prepara con harina de maíz, y se preparan los tamales. La "masa cocida" se somete a una cocción previa, con el fin de propiciar la gelatinización del almidón, y así lograr una textura más tersa (Paredes, Guevara y Pérez, 2009).

Resalta el tamizado de la masa con una manta para la eliminación máxima de residuos, y la cocción posterior, a lo que popularmente se le conoce como "masa colada". En este 
Revista Herencia, Vol. 34 (2), enero-junio, 2021.

caso, se hace una máxima extracción del almidón y, al cocinarse, se obtiene una textura firme y tersa. Algunas personas acostumbran agregar una parte de almidón de maíz o yuca para lograr una masa más blanca y suave.

También es común el uso de purés de papa o yuca, una práctica fomentada por la industria en sus campañas publicitarias y difusión de recetas para el mayor uso de la harina de maíz. Así se muestra una proporción de masa de harina y puré de papa de 1:1, y el mayor uso de grasa. Cabe mencionar que la harina de maíz repunta en Costa Rica a finales de la década de 1980.

En la cocina prehispánica, la masa no incluía grasa, y a veces ni sal, y se humedecía con agua o algún caldo, por lo que se supone que el tamal tenía una textura dura y sabor simple, en comparación con recetas después de la Colonia (Vela, 2017).

En un recetario de Costa Rica, editado en 1914, se cita el uso de sebo de res como ingrediente principal de la masa (Ramírez, 2003).

La manteca actúa como lubricante y otorga un sabor diferente. Se registran procedimientos antiguos como el batido generoso de la manteca con las manos o paletas de madera para incorporar más aire, y luego mezclarse con la masa para alcanzar una mayor suavidad. Con el pasar del tiempo, el sebo se sustituyó por manteca de cerdo, y luego por la vegetal. La proporción de masa y manteca generalmente es de 2:1, aunque hay tendencia en su reducción, principalmente a partir de la década de 1990, cuando el Ministerio de Salud y la CCSS desestimularon el uso de mantecas, principalmente la de cerdo, dado su alto contenido de ácidos grasos saturados y colesterol, debido al incremento de enfermedades crónicas no transmisibles, y se fomentó sus sustitución por aceites vegetales. Cabe mencionar que en muchos casos se registra el uso de margarina en sustitución de la manteca. 
Revista Herencia, Vol. 34 (2), enero-junio, 2021.

El éxito en la molienda, la textura final y conservación de la masa está relacionado con la humedad, la cual es determinante de la vida útil. Un cambio evidenciado es la sustitución del agua por caldo de carne o vegetales. Respecto a exaltadores de sabor, hay variantes en cuanto a la masa y las carnes. La sal, ajo y cebolla son los más comunes, seguidos por productos naturales deshidratados y pulverizados, como orégano, así como mezclas de productos naturales o saborizantes artificiales a los que popularmente se les conoce como "bomba", cuya venta es común en mercados y pulperías.

En la elaboración del tamal, la carne puede usarse cruda o cocida, aunque se prefiere cocinarla antes, con el fin de utilizar el caldo para aliñar la masa; generalmente se usan productos tales como ajo, cebolla, orégano, culantro coyote, achiote y saborizantes artificiales.

Sobre los rellenos, aún se conservan productos propios de la cocina prehispánica (Vela, 2017; Wilson, 2016), entre ellos el achiote. Los productos triturados en forma de salsas, se caracterizan por su intenso sabor y colorido, algo propio de la cocina prehispánica de Mesoamérica, y que hacia el norte de Centroamérica y sur de México son conocidas como "recados" o "pipianes". En Guanacaste el relleno de tamal se llama "guruñame" (Ross, 2020).

En Costa Rica, se pasó de una salsa espesa a vegetales en trozos de diferentes colores, grosores y formas, los cuales se colocan en el relleno crudos. Por influencia intercultural, al relleno se le agregó manteca de cerdo y trozos de tocino, así como arroz. También sobresalen hortalizas y granos traídos de Europa durante la Colonia, y la merma en el uso de otros productos propios de la cocina prehispánica, lo que muestra el dinamismo en las prácticas alimentarias. Otro aspecto que llama la atención es el uso de garbanzos y arvejas tiernas enlatadas, lo que muestra la influencia de la industria alimentaria. 
Revista Herencia, Vol. 34 (2), enero-junio, 2021.

La colocación del relleno, tal como se indicó anteriormente, tiene un sello familiar distintivo que puede variar de una comunidad a otra. Se observan variantes en cuanto a la cantidad y disposición de ingredientes en la porción de masa, y en la forma de envoltura. El relleno puede quedar visible al destapar el tamal, o bien cubierto por la masa. Es probable que la acción de sorpresa llevó al origen de la expresión ise destapó el tamal!, misma utilizada para indicar asombro o descubrimiento.

Las variantes en las recetas dependen del poder adquisitivo de las familias y estatus, algo que era común desde la prehispanidad. La variedad en ingredientes y peso final del tamal influye en el valor nutricional. Un tamal de elote o frijol puede pesar entre 75 y 150 gramos; mientras que uno de cerdo oscila entre 125 y hasta 300 gramos. Ulate y Murillo (1985), en la primera Tabla de Composición de Alimentos publicada en Costa Rica, indican que un tamal de 150 gramos puede aportar hasta $244 \mathrm{Kcal}, 8.2$ gramos de proteína y 9.0 gramos de grasa. El contenido de fibra dependerá del tipo de masa, así como del tipo y cantidad de vegetales y granos.

También existen diferencias nutricionales cuando se usa harina de maíz. El enriquecimiento de la harina de maíz con hierro y ácido fólico en Costa Rica se hace desde 1999, según el Reglamento No. 28086-S. Esta medida de salud pública se constituyó en un aliado para evitar anemias nutricionales (Ministerio de Salud, 2016). Por su parte, el exceso en el uso de sal, salsas industrializadas, alimentos enlatados y saborizantes artificiales incrementan el sodio, lo que afecta el valor nutricional.

\section{La envoltura y la cocción de los tamales}

Antiguamente, era mayor el uso de hojas de la mazorca, para lo cual se utilizaban las tusas frescas o secas remojadas previamente. El uso de tusas frescas se mantiene en la actualidad para envolver principalmente los tamales de elote. En la mayoría de tamales, 
Revista Herencia, Vol. 34 (2), enero-junio, 2021.

es común el uso de hojas de diferentes tipos musáceas soasadas, preferidas por diferentes razones: son de mayores dimensiones, flexibles, resistentes; tienen un látex que actúa como un impermeabilizante, y los aceites esenciales que se liberan en el momento del soasado otorgan un aroma único y esperado en el tamal. Estas cualidades no se encuentran en otros tipos de hojas o productos de envoltura.

Entre las hojas más comunes en Costa Rica están las de banano, plátano o guineo (Musa sp.) y bijagua (Calathea lutea). Las más disponibles en supermercados son producidas en Talamanca, y corresponden a una planta musácea nativa de Filipinas, de nombre científico Musa textilis. Esta hoja se caracteriza por su mayor resistencia y extensión en superficie, lo que facilita envoltura.

Los tipos "amarras" o hilos han cambiado con el tiempo. Antiguamente era común el uso de tiras de la misma tusa, vástago, itabo (Yucca guatemalensis), burío (Heliocarpus appendiculatus) y cabuya (Furcraea cabuya). Actualmente es más común el hilo pabilo.

La forma de amarrar los tamales tiene también un sello distintivo entre las familias, donde también se recurre a utilizar tiras de colores o lazos para diferenciar los tamales según su relleno. Al finalizar el proceso, y con los sobrantes de ingredientes, se elabora el llamado “tamal tonto" (Sánchez, 2006, p. 197). Es probable que al realizarse casi al finalizar la jornada, por la cantidad de ingredientes u hojas o cansancio, se obtenga un producto diferenciado al resto, que pudiera no estar completo o con exceso de relleno, no proporcionado o envuelto con torpeza, lo que hace que se llame de esta forma.

Las principales técnicas de conservación son el hervido frecuente y la refrigeración; pocas personas refirieron el congelado.

Disponibilidad y acceso a ingredientes, lo que se dice sobre el tamal y su efecto en el consumo 
Revista Herencia, Vol. 34 (2), enero-junio, 2021.

La elaboración artesanal se hace en las casas o pequeñas fábricas. Los ingredientes básicos están disponibles durante todo el año, lo mismo que las hojas de plátano, lo cual cual facilita la tamaleada. En los mercados provinciales o molinos comunitarios se vende maíz molido durante todo el año, mientras que la harina maíz industrializada está ampliamente disponible en el mercado nacional.

Durante los meses de noviembre a diciembre, al incrementarse la demanda de ingredientes para la elaboración de tamales, es común que el comercio procure un buen abastecimiento de productos, y se facilite la compra. También sobresalen pequeñas fábricas, sodas y restaurantes de comida tradicional que distribuyen tamales de cerdo y otros durante todo el año. Sólo en Aserrí hay más de 20 tamaleras, las cuales abastecen todo el territorio nacional. Las mismas proliferan a partir de la década de 1950, y solventaron la necesidad de contar abastecimiento del producto, ante la menor participación de personas voluntarias dedicadas a su elaboración en parroquias y organizaciones (Sedó, 2011).

Tal como se indicó, existen momentos durante el año cuando el consumo se incrementa, principalmente en Semana Santa y diciembre, así como en fechas especiales asociadas con fiestas patronales o cívicas. Para demostrar el incremento del consumo en diciembre, se cita a la Escuela de Estadística (2014), instancia a cargo de la Encuesta Actualidades, donde se estimó una producción nacional de 196 millones de piñas de tamales durante el mes de diciembre, lo que equivale a un promedio de tres tamales por persona; se calculó 28 millones de tamales que las personas comparten con otras, y 19 millones que son adquiridos en el comercio local, lo que muestra la importancia de la actividad tamalera en el país.

La misma encuesta se aplicó en diciembre del 2020, donde el 57,3\% de las personas entrevistadas afirmó que el tamal iba a estar presente en la mesa familiar, y solamente el 
Revista Herencia, Vol. 34 (2), enero-junio, 2021.

29,1\% mencionó que elaboraría tamales. Esa merma obedece a que las personas que mantienen la tradición, en su mayoría, presentan dificultades económicas debido a la COVID-19 (Escuela de Estadística, 2020).

A pesar del valor simbólico asociado con la comensalidad y el disfrute, es importante señalar que percepciones negativas asociadas con los tamales y riesgos de la salud influyen en su consumo.

El consumo de tamales y la salud han sido tema de debate en las últimas décadas. Se presenta, por un lado, el alto consumo de tamales en diciembre, y por otro, el desestímulo del consumo debido que se considera un alimento rico en "calorías", debido a que de forma descalificativa se dice que es un producto de "masa". Existen mensajes generalizados en medios de comunicación masiva que el tamal es dañino para la salud por la grasa, lo que influye en la opinión pública. En contraste, también se presenta la fuerte acción del comercio para alentar a su consumo, y la exaltación por instancias del sector cultura sobre el valor cultural de la tradición tamalera.

Es de reconocer que, dependiendo de los ingredientes y proporciones, en ciertos casos será necesario hacer ajustes en las recetas o en la frecuencia de consumo. Ello significa que no es necesario caer en el extremo de eliminar el tamal de la mesa, sino poder modificar las recetas para hacerlas más balanceadas, y continuar con la tradición.

Uno de los temas que sobresalió entre las personas entrevistadas es considerar que el tamal es "pura masa", donde se presenta una dualidad de disfrute y culpa al consumir el producto. Al respecto, el $45 \%$ de personas entrevistadas afirmó no importarle subir de peso, si se trata de comer tamales en Navidad.

En relación con la información negativa sobre el tamal, según la mayoría de personas entrevistadas para el presente estudio, se indica que la misma es obtenida en los servicios 
Revista Herencia, Vol. 34 (2), enero-junio, 2021.

de salud, redes sociales y seres allegados que enfatizan en el alto contenido de "calorías" y grasa del tamal, y el riesgo de aumentar de peso o enfermarse por su consumo en exceso. Pero... ¿qué se podría considerar por excesos en el consumo?; al respecto no hay consenso. También se hace referencia a la conveniencia de preparar los tamales en casa, con el fin de mejorar su valor nutricional y velar por la inocuidad.

Al respecto señaló una persona: “a mí me fascinan los tamales, pero por la Diabetes y la presión alta ya no puedo comerlos. Sé que me hacen daño, y procuro evitarlos, aunque me duela en el alma" (M. Pérez, comunicación personal, 7 de noviembre de 2019).

Para ampliar lo anterior, se procedió a buscar información difundida sobre el tamal por parte de la CCSS, entre los meses de octubre y diciembre del período 2014 al 2020 . Es notorio el desestímulo del consumo de tamales en exceso, tal como se indica con la siguiente nota:

Me imagino que algunos ya se comieron su primer tamal de la temporada. Eso no está mal, pero sí tienen que saber que se cuenta como una comida principal y no como una merienda, pues el contenido de grasa y calorías es alto (Caja Costarricense de Seguro Social. (s.f.) Información [Grupo de Facebook]. Facebook. Recuperado el 12 de noviembre de 2014 de https://www.facebook.com/ccssdecostarica).

Para noviembre del 2017, en la campaña "Radiografía del tamal" de la CCSS, se publicó lo siguiente:

[...] ya casi es diciembre y para entonces la alimentación tica cambiará radicalmente, principalmente por un platillo muy tradicional pero que, en exceso, podría afectarle mucho su salud, hablamos... del tamal. ¿sabía que un solo tamal podría aportarle más de 500 calorías y hasta $500 \mathrm{mg}$ de sodio? (Caja Costarricense de Seguro Social. (s.f.) Información [Grupo de Facebook]. Facebook. Recuperado el 16 de noviembre de 2017 de https://www.facebook.com/ccssdecostarica).

Las reacciones del público a las campañas realizadas por la CCSS, como institución referente de salud, destacan por su preocupación y/o negación. Uno de los comentarios dice: 
Revista Herencia, Vol. 34 (2), enero-junio, 2021.

$Y$ dale [...] que nos quieren arruinar los escapismos navideños y de fin de año! Un buen tamal y una taza de café, será y ha sido el paraíso de todo costarricense. Qué querrán que comamos pavo? (Caja Costarricense de Seguro Social. (s.f.) Información [Grupo de Facebook]. Facebook. Recuperado el 12 de diciembre de 2020 de e https://www.facebook.com/ccssdecostarica).

En el 2020, los mensajes difundidos por la CCSS se asociaron con COVID-19, los excesos y los riesgos de abusar en el consumo de tamales. Se utilizó una imagen de un tamal animado con un cubrebocas, indicándose: "Pues sí, a cuidarse doble" (Caja Costarricense de Seguro Social. (s.f.) Información [Grupo de Facebook]. Facebook. Recuperado el 29 de octubre de 2020 de e https://www.facebook.com/ccssdecostarica).

Lo anterior contrasta con los esfuerzos por parte de los sectores de cultura y academia por exaltar el valor de las tradiciones alimentarias, siendo un ejemplo de ello el Proyecto EC436 de la Escuelas de Nutrición, que en reacción a los mensajes negativos o confusos sobre el tamal, en el 2018 publicó un artículo en prensa llamado "El tamal es un héroe cultural y no el villano de la nutrición", con el fin de reafirmar que este alimento forma parte del patrimonio alimentario del país, y que lejos de desinformar o desestimular su consumo, se deben desarrollar acciones integrales para su conservación, con la posibilidad de realizar ajustes en las recetas, en concordancia con una alimentación más saludable (González, 2018).

\section{La tradición tamalera y su permanencia en el tiempo}

La práctica de preparar y consumir tamales tiene una alta relevancia en la alimentación tradicional del costarricense. Tal como lo refiere González (2012), su permanencia en el tiempo se justifica dado el valor alimentario, productivo, social, económico y cultural construido alrededor de un alimento básico: el maíz. 
Revista Herencia, Vol. 34 (2), enero-junio, 2021.

Entre las razones sobre la permanencia de la tradición tamalera en el tiempo están la necesidad de reunir a la familia, y mantener vivo el legado. Su elaboración artesanal, y el seguimiento de recetas heredadas de los ancestros hace impensable, para la mayoría, una versión de tamal industrializado que logre reunir el perfil deseado, al igual que no consumirlos en Navidad, considerada como una época "tamalera". Al respecto se cita: "Diciembre sin tamales o tamales light es como poner el pasito sin Jesús" (Caja Costarricense de Seguro Social. (s.f.) Información [Grupo de Facebook]. Facebook. Recuperado el 12 de diciembre de 2020 de e https://www.facebook.com/ccssdecostarica).

Entre los aspectos que se considera han influido negativamente en la tradición, además de la imagen negativa que pudiera tenerse del alimento, están la ausencia de la persona líder de la familia para guiar el proceso. El desconocimiento de la receta, el arduo trabajo, la complejidad en la elaboración y el desinterés de las generaciones más jóvenes por aprender a prepararlos, así como la mayor facilidad de adquirirlos en el comercio local, son razones para no elaborarlos en casa.

Otro aspecto es el alto costo económico de los ingredientes, y la imposibilidad para muchos de realizar dicha inversión. El poco apego a las recetas familiares, la mayor afinidad por otro tipo de preparaciones caseras o alimentos industrializados, y razones de salud son aspectos también mencionados por las personas para dejar de consumir tamales.

Sobre la innovación en las recetas, se refirió la necesidad de simplificar procedimientos, así como cambios en las preferencias alimentarias que llevan a la modificación de las preparaciones, lo que hace que se alejen de procedimientos antiguos o sabores familiares.

\section{Conclusiones}

El tamal, como tradición alimentaria, reúne un conjunto de saberes y prácticas de alto valor cultural, socio productivo y nutricional. Su presencia en la mesa propicia el 
Revista Herencia, Vol. 34 (2), enero-junio, 2021.

encuentro con los sabores ancestrales e historia que liga a las personas a la "cultura maicera". Trasciende la necesidad material de alimentarse, para otorgar un fuerte sentido de pertenencia e identidad. Existe gran diversidad en la combinación de ingredientes y formas de elaboración, lo cual nutricionalmente constituye una fortaleza tomando en cuenta la variedad en la alimentación. Tanto los momentos de consumo y la comensalidad sobresalen también como elementos importantes que se entretejen en la tradición tamalera.

En la elaboración, como en el consumo de tamales, es posible identificar aspectos trazadores asociados con el cambio alimentario de la población costarricense; entre ellos se destacan las afectaciones en los sistemas alimentarios tradicionales, la agricultura familiar y la merma en el consumo de maíz como alimento básico. También se evidencia la presencia creciente de alimentos industrializados por sobre alimentos en su estado natural, y la pérdida de interés en cocinar mostrado en la actualidad.

La percepción sobre el valor del alimento y su vínculo con salud también sobresale como un tema para reflexionar, dada la influencia de medios y de entidades referencia que, de forma consciente o no, descalifican una tradición alimentaria; asimismo, es posible identificar la elección de preparar o consumir un alimento, en este caso el tamal, con base a razones de salud, economía o estatus. A pesar de ello, es interesante observar la sobrevivencia de la tradición alimentaria en el tiempo.

Este trabajo constituye un insumo para continuar en la investigación sobre el tema, y para el desarrollo de estrategias educativas con enfoque integral que contribuyan con la conservación y difusión del patrimonio alimentario, base de la seguridad alimentaria y nutricional del país. 
Revista Herencia, Vol. 34 (2), enero-junio, 2021.

\section{BIBLIOGRAFÍA}

Billeb de Sinibaldi A. \& Bressani, R. (2001). Características de cocción por nixtamalización de once variedades de maíz. Archivos Latinoamericanos de Nutrición, 51(1), 86-94.

Bourges H. \& Vargas L. (2015). La cocina tradicional y la salud. Rev. Digital Universitaria UNAM, 16(5). http://www.revista.unam.mx/vol.16/num5/art36/

Bustamante, C. (Ed.). (1829). Historia general de las cosas de la Nueva España que en doce libros y dos volúmenes escribió el R.P.FR. Bernardino de Sagahún, de la observancia de San Francisco, y uno de los primeros predicadores del Santo Evangelio en aquellas regiones. México: Imprenta Alejandro Valdés. http://cdigital.dgb.uanl.mx/la/1080012524_C/1080012524_T1/1080012524_M A.PDF

Costa Rica. Ministerio de Salud. (2016, 12 de julio). Costa Rica intensifica fortificación de alimentos. https://www.ministeriodesalud.go.cr/index.php/noticias/noticias2016/963-costa-rica-intensifica-fortificacion-de-alimentos. 
Revista Herencia, Vol. 34 (2), enero-junio, 2021.

Escuela de Estadística. (2014). Encuesta Actualidades 2014. San José: Escuela de Estadística de la Universidad de Costa Rica.

Escuela de Estadística. (2020). Encuesta Nacional sobre COVID-19. San José: Escuela de Estadística de la Universidad de Costa Rica.

Fernández de Oviedo, G. (1950). Sumario Historia general y natural de las Indias. México: Fondo de Cultura Económica.

Garro, L. (2010). Saberes y sabores de Boruca. San José: Gama Print.

González, R. (2012). La alimentación tradicional en Costa Rica: propuestas para su revitalización. San José: Programa Conjunto de Políticas Interculturales para la inclusión y generación de oportunidades/Ministerio de Salud.

González, R. (2018). El tamal: héroe de la cultura o ¿villano de la nutrición?, 20 de diciembre 2016.

Diario

Extra. https:www.diarioextra.com/Noticia/detalle/319172/el-tamal:-heroe-de-lacultura-y-villano-de-la-nutricion 
Revista Herencia, Vol. 34 (2), enero-junio, 2021.

Jara, C. (1988). El léxico de origen indígena en la norma culta de San José. Revista de Filología y Lingüística, XVI(1), 109-124.

Merlo E., Bourges H., Linares E., Bye R., Mota C., Aragon F., Vargas L., Miramontes S., Carmona O. y Morán F. (2016). El maíz, nuestro rostro, nuestro corazón. México: Editorial Fundación Herdez.

Oficina Nacional de Semillas. (s.f.). Evolución del cultivo de maíz en Costa Rica. http://ofinase.go.cr/certificacion-de-semillas/certificacion-de-semillas-demaiz/evolucion-cultivo-maiz/

Paredes, O., Guevara F., Bello L. (2009). La nixtamalización y el valor nutritivo del maíz. Rev. Ciencias UNAM, 92-93.

Ramírez de Aragón, J. (2003). La cocina costarricense de 1914. San José: Editorial UCR. Ramos, Y. (2014). Así se come en Tlaxcala. Serie Cocina Indígena y Popular, No. 62. México: Consejo Nacional para la Cultura y las Artes/Instituto Nacional de Antropología e Historia/Gobierno del Estado de Tlaxcala.

Rodríguez, H. (2002). El tamal peruano, acercamiento a la realidad actual. Rev. Investigaciones Sociales Universidad San Martín de Porres, VI(10), 9-22. 
Revista Herencia, Vol. 34 (2), enero-junio, 2021.

Ross, M. (1990). Al calor del fogón. 500 años de cocina costarricense. San José: Editorial Farben.

Ross, M. (29 de diciembre de 2020). Tamales! [Mensaje en un blog]. Recuperado de https://sinreservas.cr/tamales/

Sagahún, Bernardino Fray. (1989). Historia general de las cosas de la Nueva España, Libro X. México: Consejo Nacional para la Cultura y las Artes.

Sánchez, V. (2006). Léxico relativo al ámbito del tamal en Costa Rica. Káñina, XXX(2), 191-197.

Sedó, P. (2011). El turno: Un acercamiento a este espacio festivo y de expresión cultural y gastronómica en comunidades del Valle Central de Costa Rica. San José: Escuela de Nutrición de la Universidad de Costa Rica.

Ulate, E., Murillo S. (1985). Tabla de composición de alimentos para Costa Rica. San José: Instituto de Investigaciones en Salud, Universidad de Costa Rica. 
Revista Herencia, Vol. 34 (2), enero-junio, 2021.

Vela, E. (2017). El tamal en México. Breve historia. Rev. Arqueología Mexicana, edición especial octubre $76,8-21$.

Wilson, C. (2016). El poyo, la piedra y el nixtamal: cocina al estilo de Guatemala para neófitos. Guatemala: Serviprensa S.A. 
Revista Herencia, Vol. 34 (2), enero-junio, 2021. 